\title{
A Brain-Kidney Connection: The Delicate Interplay of Brain and Kidney Physiology
}

\author{
William D. Freeman • Hani M. Wadei
}

Published online: 12 February 2015

(c) Springer Science+Business Media New York 2015

The central nervous system (CNS) and kidneys are strongly interconnected. Afferent impulses from the CNS regulate renal blood flow, glomerular filtration rate (GFR), and affect renal sodium handling [1]. Vasopressin hormone is secreted from the posterior pituitary gland and acts on the collecting ducts of the distal nephron to regulate water balance and serum osmolality. However, the kidney is not a target organ that solely responds to impulses from the CNS. Impulses originating from the kidney are carried via unmyelinated and thinly myelinated fibers to the CNS and the contralateral kidney to regulate CNS activity and coordinate renal sodium handling with the contralateral kidney. In animal models with acute kidney injury (AKI), the levels of inflammatory cytokines increase in the brain with evidence of anatomic and functional brain injuries [2]. Both organs also share a common feature-a tight autoregulatory mechanism that maintains constant blood flow over a wide range of blood pressures. It is unclear, however, whether both the renal and brain autoregulatory mechanisms are interconnected in such a way that changes that occur in one organ affect the blood flow in the other.

In this issue of Neurocritical Care, two articles by two separate NeuroICU teams report some important observations on the connection between the brain and kidney vascular beds and autoregulatory mechanisms. The first article by Dias et al. [3] reports a retrospective small series of $18 \mathrm{TBI}$ patients in which various laboratory values were

\section{W. D. Freeman $(\square)$}

Departments of Neurosurgery, Neurology and Critical Care, Mayo Clinic, 4500 San Pablo Rd, Jacksonville, FL 32224, USA e-mail: freeman.william1@mayo.edu

H. M. Wadei

Department of Transplantation, Mayo Clinic, Jacksonville, FL 32224, USA analyzed, including serum creatinine. The authors estimated both creatinine clearance (CrCL) and GFR using two creatinine-based equations: the Cockroft-Gault and the four-point Modification of Diet in Renal Diseases (MDRD) equations, respectively. ICP recording with mean arterial pressure (MAP) via a multimodal monitoring (MMM) computer was obtained, and the authors utilized the MMM data to acquire pressure-reactivity index (PRX) as a surrogate for cerebrovascular auto regulation (CVAR) and correlated PRX with CrCL and GFR, as well as other physiological and laboratory parameters. The authors' data suggest that patients with brain injuries with better CVAR as measured by PRX were statistically associated with renal hyperfiltration, augmented renal clearance (ARC), and potentially better outcomes. While these findings are intriguing, the results should be interpreted with great caution. First, this study was not intended to monitor GFR, and therefore no "gold standard" method for GFR measurement, such as continuous infusion of iothalamate, was utilized. Second, intravenous fluid infusion in critically ill patients and mannitol use in TBI patients will affect serum creatinine concentration without any meaningful change in kidney function [4]. Third, although using creatinine-based equations to calculate $\mathrm{CrCl}$ (in the case of $\mathrm{CG}$ ) and GFR (in the case of MDRD) seems appealing, in reality, serum creatinine is the only variable in these equations that demonstrates day-to-day changes, while the remaining variables in the equation such as age, gender, and race will remain constant. In addition, it is unclear whether ideal body weight, admission weight, or daily weight was utilized in calculating $\mathrm{CrCl}$ by $\mathrm{CG}$. MDRD is intended for chronic kidney disease (CKD) patients and is biased in patients without CKD. Because of the way "hyperfiltration" was actually measured and reported, the whole idea of ARC should be taken with a grain of salt. A fourth 
limitation of this study is the inability to prove a cause-andeffect relationship, and it is unclear whether patients with maintained kidney function have intact brain autoregulation or vice versa. There is no mention in the paper of whether any of the patients experienced abdominal compartment syndrome (ACS) as a result of their original trauma, or had concurrent kidney, pelvic, or flank trauma. As observed in the cerebral circulation, increased intraabdominal pressure in ACS is associated with reduced GFR and increased serum creatinine. Finally, even minor elevations in serum creatinine are associated with increased in-hospital mortality in all currently studied groups of patients, including those with TBI. Therefore, the authors' observation that serum creatinine elevation was associated with higher mortality should not be surprising [5]. Nevertheless, despite these limitations, this study is an extension of previously published evidence that cerebral perfusion and GFR are interconnected, with patients who experience cerebral hypoperfusion at an increased risk of renal injury and vice versa, with subsequent effects on outcome and mortality [6, 7]. Without a larger study, control population, or similar validation, the clinical impact of this study remains to be seen outside the realm of physiological insights.

The second article by Osgood et al. [8] provides a completely different insight into "brain-kidney" connections because it examines the effect of renal replacement therapy (RRT) on acute brain herniation in brain-injured patients. The authors describe a series of four patients, all of whom had RRT with subsequent worsening or de novo brain edema, herniation, and neurological deterioration, or death. Of note, one of the patients had a hemicraniotomy and still developed brain herniation. The authors graphically outline serum sodium, BUN, and bicarbonate over time with RRT, which are important laboratory factors in the plasma (Serum) osmolality equation (Posm) Fig. 1. Each of the cases that deteriorated to death had a relative downward shift in Posm from a higher to lower state (Fig. 1), either from reduced relative sodium or BUN values. The four cases presented in this series highlight different mechanisms of brain edema that can result in patients receiving RRT. In the first two cases, both a rapid drop in BUN and dangerous rapid decline in serum sodium (almost $15 \mathrm{mEq} / \mathrm{L}$ drop in serum $\mathrm{Na}$ level in case 1 over a 15-h period) contributed to the brain edema and herniation. Case 3 is rather interesting, as the BUN and sodium levels remained stable over 2 days, but the serum bicarbonate, which started to rise prior to the RRT initiation, had almost doubled at time of brain herniation and could have contributed to the brain edema via inducing paradoxical reduction in the CSF $\mathrm{pH}$. The fourth case describes brain herniation in a patient on continuous RRT (CRRT), which is extremely rare. The slow decline in serum sodium and BUN levels, which are typical for CRRT, suggests that another cause of brain edema such as hypoxia or vasogenic edema should be ruled out. Irrespective of the cause of cerebral edema, this article highlights that patients with brain injury who need RRT should be dialyzed with

\section{Brain-Kidney and Plasma Osmolality States}

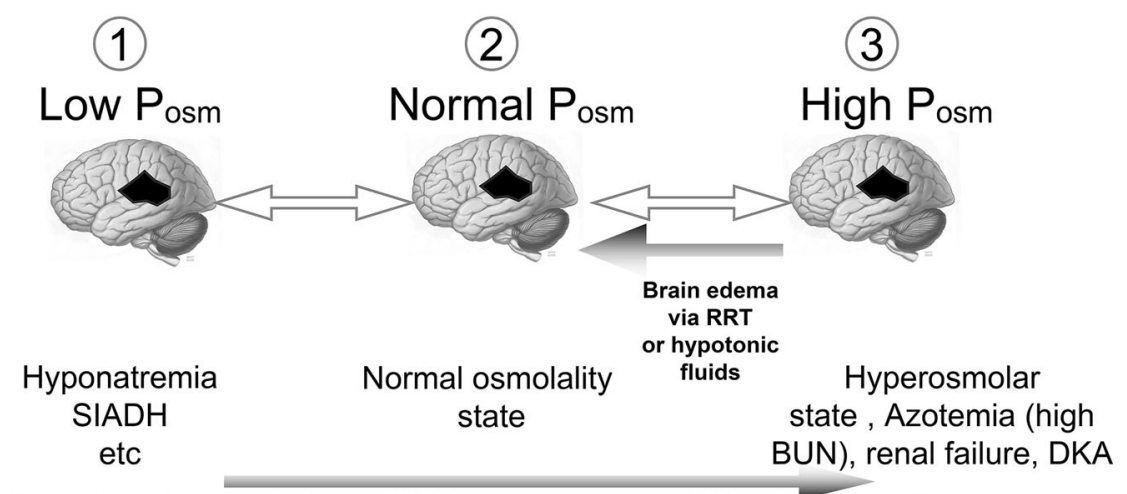

Central pontine myelinolysis (CPM) can occur acute shifting from state $1 \longrightarrow 3$

(overcorrection of serum sodium or $\mathrm{P}_{\mathrm{osm}}$ from acute hyperglycemia treatment (hypertonic insult) ${ }^{11}$ )

Fig. 1 Brain-kidney and osmolality state considerations. The figure demonstrates three broad states of plasma/serum osmolality (Posm): low, normal, or high. Changes between Posm states can cause brain injury. For example, a subacute/chronic hypotonic/hyponatremic patient who is overcorrected (relative hypertonic insult) to a normal serum sodium (e.g., >12meQ/24 h) may develop CPM. Similarly, patients who are hyperosmolar such as diabetic ketoacidosis (DKA) if dropped too quickly may develop global brain edema. Authors
Osgood et al. report cases of relative Posm correction either by sodium or urea reduction rate similarly causing brain edema, herniation, and death. This may be due to intracellular (neuronal) osmolality being subacutely elevated (i.e., idiogenic osmole hypothesis) relative to serum and interstitial space, which causes relative water influx of those neurons due to a rapid osmolality change toward normal range 
extreme caution with special care directed to brain CT scan findings of edema, as well as dialysate composition and blood urea level. The article raises important questions about potentially modifiable factors to reduce brain edema and herniation such as the dialysis rate, the dialysate sodium level, or the urea reduction ratio (URR), which may not be routinely monitored by nephrologists for brain-injured patients. Also inherent to this physiology is the understanding that plasma osmolality is more than just sodium and BUN and includes glucose. In a prior publication in this journal, a case of hyperglycemia (relative hypertonic insult) with rapid correction led to central pontine myelinolysis (CPM) [9].

In conclusion, the brain-kidney interconnection, as both groups point out, is more complicated than can be explained by examining either organ independently, and it likely involves inflammatory, unknown hormonal, or cytokinemediated organ dysfunction, genetic, and blood pressurerelated physiology. Therefore, the above two articles provide important contributions to the field of neurocritical care in terms of "cerebro-nephrology" inter-relationships, but they must also be understood in the greater context of critical care, molecular/cellular biology, medicine, and physiology.

Conflict of interest Drs. Freeman and Wadei report no conflicts of interest or disclosures with any pharmaceutical or device industry, nor any financial relationships with regards to this work.

\section{References}

1. DiBona GF. Physiology in perspective: the Wisdom of the Body. Neural control of the kidney. Am J Physiol Regul Integr Comp Physiol. 2005;289:R633-41.

2. Liu M, Liang Y, Chigurupati S, et al. Acute kidney injury leads to inflammation and functional changes in the brain. J Am Soc Nephrol. 2008;19:1360-70.

3. Dias C, Gaio AR, Monteiro E, et al. Kidney-brain link in traumatic brain injury patients?. Neurocrit Care: A preliminary report; 2014.

4. Macedo E, Bouchard J, Soroko SH, et al. Fluid accumulation, recognition and staging of acute kidney injury in critically-ill patients. Crit Care. 2010;14:R82.

5. Rewa O, Bagshaw SM. Acute kidney injury-epidemiology, outcomes and economics. Nat Rev Nephrol. 2014;10:193-207.

6. Ono M, Arnaoutakis GJ, Fine DM, et al. Blood pressure excursions below the cerebral autoregulation threshold during cardiac surgery are associated with acute kidney injury. Crit Care Med. 2013;41:464-71.

7. O'Rourke MF, Safar ME. Relationship between aortic stiffening and microvascular disease in brain and kidney: cause and logic of therapy. Hypertension. 2005;46:200-4.

8. Osgood M, Compton R, Carandang R, Hall W, Kershaw G, Muehlschlegel S (2014) Rapid unexpected brain herniation in association with renal replacement therapy in acute brain injury: caution in the neurocritical care unit. Neurocrit Care.

9. Burns JD, Kosa SC, Wijdicks EF. Central pontine myelinolysis in a patient with hyperosmolar hyperglycemia and consistently normal serum sodium. Neurocrit Care. 2009;11:251-4. 